\title{
SOSTENIBILIDAD UNIVERSITARIA CON ENFOQUE EN LA EDUCACIÓN AMBIENTAL: REDES DE COLABORACIÓN Y CLÚSTER TEMÁTICOS DE LA PRODUCCIÓN CIENTÍFICA MUNDIAL
}

Aníbal Enrique Toscano Hernández Universidad del Sinú Colombia

Deivi David Fuentes Doria Universidad Pontificia Bolivariana Colombia

Melisa Andrea Fajardo Pereira Universidad Internacional Iberoamericana Puerto Rico 
Aníbal Enrique Toscano Hernández

Deivi David Fuentes Doria

Melisa Andrea Fajardo Pereira

JEL: A10, A12, A19

\title{
Sostenibilidad universitaria con enfoque en la educación ambiental: redes de colaboración y clúster temáticos de la producción científica mundial
}

\section{Resumen}

El objetivo de este artículo es caracterizar la producción científica mundial relacionada a la sostenibilidad y educación ambiental en la universidad. La metodología aplicada se sustenta en la identificación y análisis de publicaciones de revistas científicas indexadas en la base de datos Scopus, junto con la utilización del software conocido como VOSviewer que facilita la visualización de mapas de red basados en datos bibliográficos, lo cual resulta en un aporte significativo y novedoso en este campo de estudio. En efecto, los resultados reflejan un importante incremento registrado en el número anual de publicaciones, al registrar un incremento del 1400\% de publicaciones entre 1984 y 2017. En lo referente a la dispersión en el campo de estudio existe en la producción científica revistas de impacto mundial, las principales 14 revistas concentran el $44,6 \%$ de las publicaciones y acumulan el 51,1\% de las citaciones. En conclusión, los hallazgos permiten visualizar las características generales, redes de colaboración, visibilidad e influencia, y las principales tendencias temáticas de la producción científica relacionada con la sostenibilidad y la educación ambiental en la universidad.

Palabras clave: Sostenibilidad, desarrollo sostenible, educación superior

Durabilité des universités en mettant l'accent sur l'éducation environnementale: réseaux de collaboration et groupes thématiques de la production scientifique mondiale

\section{Résumé}

L'objectif de cet article est de caractériser la production scientifique mondiale liée à la durabilité et à l'éducation environnementale à l'université. La méthodologie appliquée est basée sur l'identification et l'analyse des publications de revues scientifiques indexées dans la base de données Scopus, ainsi que sur l'utilisation d'un logiciel connu sous le nom de VOSviewer qui facilite la visualisation des cartes du réseau basées sur des données bibliographiques, ce qui se traduit par une contribution importante et nouvelle dans ce domaine d'étude. En effet, les résultats reflètent une augmentation significative enregistrée dans le nombre annuel de publications, enregistrant une augmentation de $1400 \%$ des publications entre 1984 et 2017. Concernant la dispersion dans le domaine d'étude, il existe des magazines à impact mondial dans la production scientifique, les 14 principaux magazines concentrent $44,6 \%$ des publications et cumulent $51,1 \%$ des citations. En conclusion, les résultats nous permettent de visualiser les caractéristiques générales, les réseaux de collaboration, la visibilité et l'influence, ainsi que les principales tendances thématiques de la production scientifique liées à la durabilité et à l'éducation environnementale à l'université.

Mots clés: Durabilité, développement durable, enseignement supérieur.

\section{University sustainability with a focus on environmental education: collaboration networks and thematic clusters of world scientific production}

\begin{abstract}
The objective of this article is to characterize the world scientific production related to sustainability and environmental education at the university. The applied methodology is based on the identification and analysis of scientific journal publications indexed in the Scopus database, together with the use of software known as VOSviewer that facilitates the visualization of network maps based on bibliographic data, which results in a significant and novel contribution in this field of study. In effect, the results reflect a significant increase registered in the annual number of publications, registering an increase of $1400 \%$ of publications between 1984 and 2017. Regarding the dispersion in the field of study, there are world-impact magazines in the scientific production, the main 14 magazines concentrate $44.6 \%$ of the publications and accumulate $51.1 \%$ of the citations. In conclusion, the findings allow us to visualize the general characteristics, collaboration networks, visibility and influence, and the main thematic trends of scientific production related to sustainability and environmental education at the university.
\end{abstract}

Keywords: Sustainability, sustainable development, higher education. 


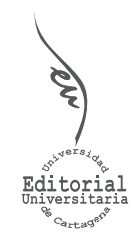

Artículo de Investigación

\section{Sostenibilidad universitaria con enfoque en la educación ambiental: redes de colaboración y clúster temáticos de la producción científica mundial}

INFORMACIÓN DEL ARTÍCULO

Recepción del artículo: 01/03/2018

Concepto de evaluación: 17/04/2018

Aceptación del artículo: 03/06/2018
Aníbal Enrique Toscano Hernández*
Universidad del Sinú, Colombia

Deivi David Fuentes Doria Universidad Pontificia Bolivariana, Colombia

Melisa Andrea Fajardo Pereira Universidad Internacional Iberoamericana, Puerto Rico

\section{INTRODUCCIÓN}

Hoy en día, el medio ambiente de nuestro planeta está registrando un cambio rápido, arrollador, duradero y sin precedentes que está promoviendo profundas modificaciones en las estructuras sociales y económicas a nivel mundial (UNESCO, 2014). De hecho, dado el carácter limitado de los recursos naturales disponibles en nuestro planeta, en la literatura académica se evidencian múltiples y constantes esfuerzos por mejorar los parámetros, métodos, metodologías y teorias que fomenten el uso sostenible de estos recursos (Vergara-Arrieta \& Álvarez-Carval, 2017). De esta manera, la sostenibilidad se ha consolidado como un concepto ampliamente utilizado en todo el mundo y que supone admitir que el planeta posee recursos limitados, exigiendo planteamientos holísticos y globales para enfrentar los problemas a los que se enfrenta la humanidad (Vilches \& Gil, 2012).

En este contexto, las organizaciones de todo tipo han comenzado a introducir o cambiar políticas, estrategias y comportamientos para afrontar asuntos impostergables como la contaminación y la exploración de recursos (Linnenluecke \& Griffiths, 2013). En el caso de las universidades, Tilbury (2010) menciona que la sostenibilidad debe formar parte de los principios básicos de la gestión de la educación superior y debe influir en el desarrollos de las principales actividades misionales de las universidades. En efecto, en la última década, un creciente número de universidades han promovido diversas

*Autor de correspondencia: anibalenriquetoscano@gmail.com 
iniciativas orientadas a integrar la sostenibilidad en sus actividades misionales de docencia e investigación (Lazzarini, Pérez-Foguet, \& Boni, 2018).

Sin embargo, a pesar de la creciente cantidad de universidades que se han comprometido con iniciativas de sostenibilidad, la mayoría continúan gestionándose tradicionalmente (Gaudiano, Meira-Cartea, \& Martínez-Fernández, 2015). Al respecto, se debe considerar que, para las universidades, "de la pertinencia de su misión y de la praxis de su visión está su participación como protagonista o como simple espectadora adaptable al cambio" (Herrera-Llamas, 2014, p. 235). Por lo que, en muchos casos, las universidades se encuentran rezagadas en su contribución a la sostenibilidad de la sociedad, en comparación con organizaciones empresariales y gobiernos (Lozano, Lukman, Lozano, Huisingh, \& Lambrechts, 2013).

Frente a este contexto, el enorme interés por el estudio de la sostenibilidad en el contexto universitario han promovido el crecimiento de la producción científica en este campo de estudio en los últimos años (Schmitt Figueiró \& Raufflet, 2015; Toscano-Hernández, Álvarez-González, \& Sanzo-Pérez, 2018). Entre las principales tendencias temáticas de la investigación científica relacionadas con la sostenibilidad en la educación superior se pueden mencionar tres (Toscano-Hernández et al., 2018): (1) la educación para la sostenibilidad, (2) el rol de la universidad en relación a iniciativas hacia el desarrollo sostenible, y (3) la gestión de la sostenibilidad en la educación superior. En el caso de la educación para la sostenibilidad, esta implica fomentar cambios efectivos en la comprensión del proceso educativo, a pesar que desde la perspectiva tradicional es entendida como la introducción de contenidos ambientales en los currículos(Karatzoglou, 2012; Schmitt Figueiró \& Raufflet, 2015). Dado que difícilmente se puede lograr ofrecer buena educación ambiental en la universidad si no va a aparejada por la gestión adecuada de los aspectos ambientales, la educación ambiental debe ir relacionada con prácticas de gestión sostenible en la universidad (Alba, D., Alonso, I. y Benayas, 2011).

Ahora bien, considerando el potencial de la universidad para impulsar el crecimiento económico y la productividad (World Bank, 2017), el estudio de la sostenibilidad en la universidad en general, y la educación ambiental en particular, tiene la oportunidad de contribuir significativamente a la transformación hacia una sociedad global más sostenible y equitativa (Lazzarini et al., 2018). En este sentido, dado el crecimiento de la producción científica relacionada (Alba Hidalgo, 2017; Karatzoglou, 2012; Schmitt Figueiró \& Raufflet, 2015), las síntesis de múltiples investigaciones resultan significativamente útiles como herramientas eficaces para acceder a los recientes desarrollos de la investigación en este campo de estudio (Toscano-Hernández et al., 2018). Tal es el caso de los estudios bibliométricos que, como metodología de investigación del campo de meta-estudios reflexivos de la literatura, facilitan obtener una visión general de las estructura intelectual y valiosas evaluaciones de las tendencias de campos de estudio (Pipere, Veisson, \& Salite, 2015).

En este sentido, en la literatura académica se encuentran diversos estudios que utilizan herramientas bibliométricas para analizar la producción científica relacionada con la sostenibilidad en el contexto universitario, desde diversas perspectivas y enfocado en diferentes contextos. En concreto, entre los estudios bibliométricos se puede resaltar como éstos abordan la sostenibilidad universitaria de forma general (ToscanoHernández et al., 2018), mientras otros se preocupan por la educación docente para la sostenibilidad (Pipere et al., 2015) y las características de las comunidades universitarias involucradas en el fomento del desarrollo humano sostenible (Lazzarini \& Pérez-Foguet, 
2018; Lazzarini et al., 2018). Sin embargo, con base en la exploración de la literatura académica, ninguna publicación anterior ha abordado el panorama mundial de la investigación científica relacionada con la sostenibilidad en la universidad con enfoque en la educación ambiental a partir de un analisis bibliométrico.

En general, a partir de lo descrito anteriormente, el objetivo de este trabajo es complementar los estudios bibliométricos anteriores, al presentar los resultados de un análisis bibliométrico de la investigación científica en sostenibilidad y educación ambiental en la universidad. La metodología del presente trabajo se sustenta en publicaciones de revistas científicas indexadas en la base de datos Scopus, junto con la utilización del software conocido como VOSviewer que facilita la visualización de mapas de red basados en datos bibliográficos (Perianes-Rodriguez, Waltman, \& Van Eck, 2016), lo cual resulta en un aporte significativo y novedoso en este campo de estudio.

\section{METODOLOGÍA}

En el trabajo se planteó el procedimiento, a partir de los principios de revisión sistemática de literatura desarrollados por Tranfield, Denyer, \& Smart (2003), que se describe en este apartado. Estos ítems son resultado de un consenso razonable que ha surgido en cuanto a las características metodológicas deseables para reportar los resultados de trabajos de revisión bibliográfica basados en pasos replicables y transparentes (Moher et al., 2009; Tranfield et al., 2003).

\section{Fuente de información}

En este trabajo se utiliza la base de datos Scopus de Elsevier como fuente de búsqueda de información, dado que permite garantizar la cobertura más amplia en nuestro estudio bibliométrico y minimizar el sesgo que origina la búsqueda de información en bases de datos específicas, al ser consideradas las bases más completas, con estándares de indexación más exigentes y una poderosa capacidad de análisis de citaciones (Bartels, 2013; Falagas, Pitsouni, Malietzis, \& Pappas, 2008). Adicionalmente, esta base de datos registra el indicador SCImago Journal Rank (SJR), que permite evidenciar la visibilidad de las revistas científicas indexadas desde 1996.

\section{Estrategia de búsqueda}

Considerando los objetivos del presente trabajo, se consideran documentos de producción científica publicados en fuentes indexadas en Scopus. Se opta por limitar la búsqueda de producción científica publicada entre el año 1984 y 2017.

Las expresiones de búsquedas fueron escogidas al considerar que los términos "sustanaible", "university" y "environmental education", y sus equivalentes, están siendo utilizando de formas alternativas en la literatura científica. Se realiza la búsqueda en títulos, resúmenes y palabras claves de las publicaciones utilizando la siguiente expresión de búsqueda: (TITLE-ABS-KEY (sustainable OR sustainibility OR sustainable development) AND TITLE-ABS-KEY (university OR universities) AND TITLE-ABS-KEY (environmental education)). El resultado de la aplicación de la 
estrategia de búsqueda descrita anteriormente dio lugar a la identificación inicial de un total 301 referencias de publicaciones relacionadas con el estudio de la sostenibilidad y la educación ambiental en la universidad.

\section{Identificación y selección de estudios}

Se identificaron y seleccionaron las referencias que cumplieran los siguientes criterios: (1) únicamente artículos de investigación o revisiones de literatura; (2) publicados en revistas científicas clasificadas en el SJR (SCImago Journal Rank); (3) publicados entre 1984 y 2017. Con la aplicación los criterios de selección anteriores que garantizan la identificación de publicaciones de alto impacto y visibilidad científica, se logró la identificación de 231 referencias de los estudios científicos de mayor relevancia publicados en el periodo comprendido entre 1984 y 2017.

\section{Recopilación y análisis de datos}

La recopilación de los datos de las referencias relacionadas permite realizar una caracterización cuantitativa que considera los siguientes criterios: (1) año de publicación, (2) revistas, y (3) número de citaciones registradas hasta junio de 2018. Mientras que el análisis de la producción científica referenciada, como se desarrollará más adelante en este trabajo, permite visualizar la complejidad y variedad de los hallazgos. Ante esta situación, los mapas de red basados en datos bibliográficos, conocidos como mapas bibliométricos, son especialmente útiles en la identificación de grupos de elementos relacionados entre las publicaciones científicas (Perianes-Rodriguez et al., 2016; Van Eck \& Waltman, 2010).

Ahora bien, considerando la funcionalidad del software VOSviewer para la visualización de mapas bibliométricos de una manera fácil de interpretar (Perianes-Rodriguez et al., 2016), a partir de los descrito por Van Eck \& Waltman (2016) se construyen y analizan los mapas de red de los principales datos bibliográficos de la producción científica relacionada con la sostenibilidad en el contexto universitario desde la perspectiva de la educación ambiental utilizando: (1) los análisis de tipo co-autoría, citaciones y co-ocurrencia; (2) las unidades de análisis relacionadas con los autores, organizaciones, países y palabras claves; y (3) las visualizaciones de clúster y temporal.

\section{Reporte de resultados}

Ahora bien, el reporte de los resultados del análisis de las 231 referencias identificadas y seleccionadas se desarrolló en tres partes: (1) análisis descriptivo, (2) redes de colaboración, (3) visibilidad e influencia y (4) tendencias temáticas.

Inicialmente, en el análisis Descriptivo, se realiza una descripción general de la producción científica a partir de los criterios de caracterización cuantitativa definidas en el sub-apartado anterior. Seguidamente, en redes de colaboración, se presentan los resultados del análisis de co-autoría de los autores, afiliaciones y países. Posteriormente, en visibilidad e influencia, se muestran los resultados del análisis de citaciones valorando como unidad de observación los autores, organizaciones y países; así como los valores de SJR de las revistas y el índice h de las publicaciones. Finalmente, en Tendencias Temáticas, se presentan los resultados del análisis de co-ocurrencia de palabras claves de las publicaciones relacionadas. 


\section{RESULTADOS}

Los resultados de aplicar la estrategia de búsqueda inicial, tal como se ha anticipado en el apartado anterior, permitió identificar un total de 301 referencias de publicaciones relacionadas con la sostenibilidad y la educación ambiental en la universidad. Estos resultados incluyen diversos tipos de publicaciones como: libros, capítulos de libro, documentos de conferencias, material de editorial, artículos científicos, entre otros.

Sin embargo, entre las referencias de publicaciones científicas identificadas con la estrategia de búsqueda inicial, fueron seleccionadas las 231 referencias de las publicaciones de mayor relevancia de acuerdo a los criterios descritos en el apartado de Identificación y Selección de Estudios de la metodología de este documento.

\section{Análisis descriptivo}

En este apartado, con el propósito de evidenciar la evolución cuantitativa de las publicaciones científicas relacionadas con la sostenibilidad y la educación ambiental en la universidad, se presenta un análisis general de 231 referencias de las publicaciones de mayor relevancia.

En la Figura 1, que presenta la evolución temporal en el número de publicaciones identificadas, se refleja el importante interés generado en este campo de estudio. El número anual de publicaciones evidencia un incremento importante en el periodo bajo estudio, al pasar de 1 a 15 publicaciones entre 1984 y 2017, lo cual representa un incremento del $1400 \%$ en el número de documentos anuales. Mientras que, al analizar las publicaciones por año, se obtiene un promedio de 8,8 referencias por año.

Figura 1. Evolución temporal de publicaciones científicas relacionadas con la sostenibilidad universitaria con enfoque en la educación ambiental. 1984-2017.

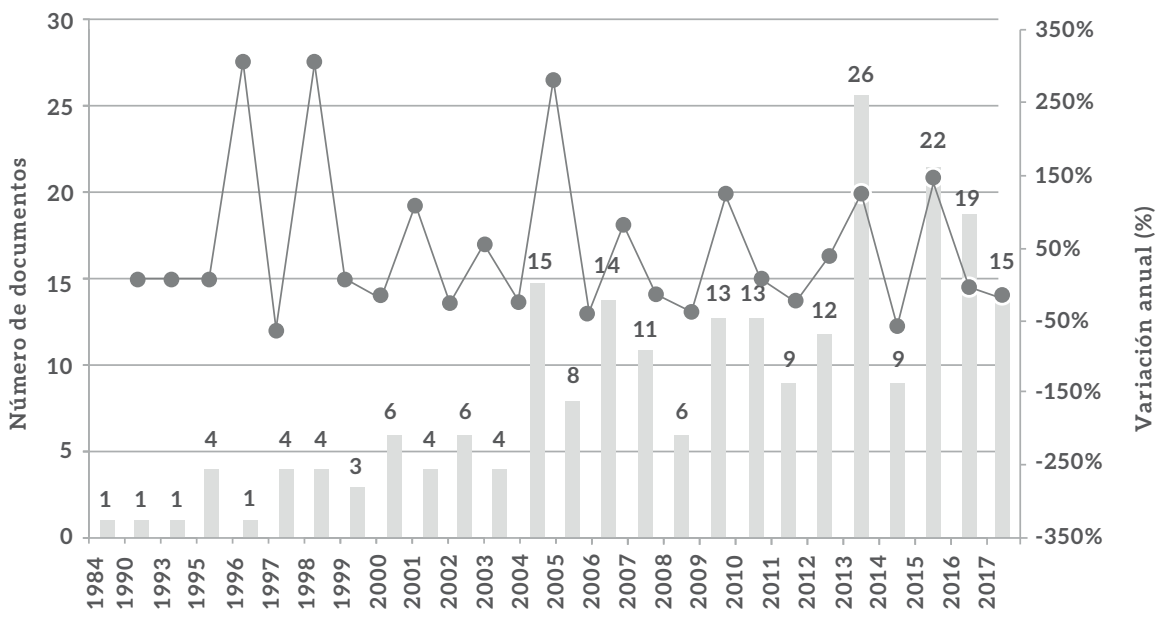

Número de documentos $\longrightarrow$ Variación Anual

Fuente: Elaboración propia a partir de información de Scopus. 
En el periodo bajo observación, el mayor crecimiento anual en el número de publicaciones se registra en los años 1995 y 1997 con un aumento del 300\%, en relación al año inmediatamente anterior, seguidamente se encuentran los años 2004 (275\%), 2015 (144\%), 2009 (117\%) y 2013 (117\%). En otro sentido, se observa como aquellos años en los que se registra la mayor reducción en el número de publicaciones anuales con respecto al año inmediatamente anterior fueron 1996 (-75\%), 2014 (-65\%), 2005 (47\%) y 2008 (-45\%). Se debe señalar, como a partir de 2013, el año que registra el mayor número de publicaciones, se observa una disminución progresiva de numero de estudios en este campo.

Los resultados muestran como las publicaciones científicas sobre la sostenibilidad y la educación ambiental en la universidad, que incluye publicaciones entre los años 1984 y 2017, se distribuyen en 124 revistas y registran un total de 1467 citaciones (ver Tabla 1).

Tabla 1.

Distribución de publicaciones científicas relacionadas con la sostenibilidad universitaria con enfoque en la educación ambiental, por revistas y citaciones. 1984-2017.

\begin{tabular}{|c|c|c|c|c|c|}
\hline \multirow{2}{*}{ Revistas (país) } & \multirow{2}{*}{$\begin{array}{l}\text { Cuartil } \\
\text { SJR }\end{array}$} & \multicolumn{2}{|c|}{ Documentos } & \multicolumn{2}{|c|}{ Citación } \\
\hline & & $\mathbf{N}^{\circ}$ & $\%$ & $\mathrm{~N}^{\circ}$ & $\%$ \\
\hline $\begin{array}{l}\text { Australian Journal of Environmental } \\
\text { Education (Reino Unido) }\end{array}$ & Q2 & 25 & $10.8 \%$ & 173 & $11.8 \%$ \\
\hline Sustainability (Suiza) & Q2 & 12 & $5.2 \%$ & 34 & $2.3 \%$ \\
\hline $\begin{array}{l}\text { International Journal of Sustainability } \\
\text { in Higher Education (Reino Unido) }\end{array}$ & Q2 & 10 & $4.3 \%$ & 185 & $12.6 \%$ \\
\hline $\begin{array}{l}\text { Journal of Cleaner Production } \\
\text { (Paises Bajos) }\end{array}$ & Q1 & 10 & $4.3 \%$ & 81 & $5.5 \%$ \\
\hline $\begin{array}{l}\text { Journal of Environmental Studies } \\
\text { and Sciences (Estados Unidos) }\end{array}$ & Q2 & 10 & $4.3 \%$ & 28 & $1.9 \%$ \\
\hline $\begin{array}{l}\text { Environmental Education Research } \\
\text { (Reino Unido) }\end{array}$ & Q1 & 5 & $2.2 \%$ & 116 & $7.9 \%$ \\
\hline $\begin{array}{l}\text { Environmental Engineering and } \\
\text { Management Journal (Rumania) }\end{array}$ & Q3 & 5 & $2.2 \%$ & 7 & $0.5 \%$ \\
\hline $\begin{array}{l}\text { International Research in Geographical } \\
\text { and Environmental Education } \\
\text { (Reino Unido) }\end{array}$ & Q2 & 5 & $2.2 \%$ & 42 & $2.9 \%$ \\
\hline $\begin{array}{l}\text { Environmental Engineering and } \\
\text { Management Journal (Rumania) }\end{array}$ & Q3 & 5 & $2.2 \%$ & 7 & $0.5 \%$ \\
\hline
\end{tabular}

Fuente: Elaboración propia a partir de información de Scopus. 
Tabla 1.

Distribución de publicaciones científicas relacionadas con la sostenibilidad universitaria con enfoque en la educación ambiental, por revistas y citaciones. 1984-2017.

\begin{tabular}{|c|c|c|c|c|c|}
\hline \multirow{2}{*}{ Revistas (país) } & \multirow{2}{*}{$\begin{array}{l}\text { Cuartil } \\
\text { SJR }\end{array}$} & \multicolumn{2}{|c|}{ Documentos } & \multicolumn{2}{|c|}{ Citación } \\
\hline & & $\mathbf{N}^{\circ}$ & $\%$ & $\mathrm{~N}^{\circ}$ & $\%$ \\
\hline $\begin{array}{l}\text { International Research in Geographical } \\
\text { and Environmental Education } \\
\text { (Reino Unido) }\end{array}$ & Q2 & 5 & $2.2 \%$ & 42 & $2.9 \%$ \\
\hline $\begin{array}{l}\text { Environmental Management } \\
\text { (Alemania) }\end{array}$ & Q1 & 4 & $1.7 \%$ & 43 & $2.9 \%$ \\
\hline $\begin{array}{l}\text { Journal of Geography in Higher } \\
\text { Education (Reino Unido) }\end{array}$ & Q2 & 4 & $1.7 \%$ & 34 & $2.3 \%$ \\
\hline Sustainability (Estados Unidos) & Q3 & 4 & $1.7 \%$ & 1 & $0.1 \%$ \\
\hline $\begin{array}{l}\text { Applied Environmental Education } \\
\text { and Communication (Reino Unido) }\end{array}$ & Q4 & 3 & $1.3 \%$ & 1 & $0.1 \%$ \\
\hline $\begin{array}{l}\text { International Journal of Environment } \\
\text { and Sustainable Development } \\
\text { (Reino Unido) }\end{array}$ & Q4 & 3 & $1.3 \%$ & 4 & $0.3 \%$ \\
\hline $\begin{array}{l}\text { International Review of Education } \\
\text { (Paises Bajos) }\end{array}$ & Q2 & 3 & $1.3 \%$ & 1 & $0.1 \%$ \\
\hline Otras 110 revistas & - & 128 & $55.4 \%$ & 717 & $48.9 \%$ \\
\hline TOTAL GENERAL & & 231 & $100 \%$ & 1467 & $100 \%$ \\
\hline
\end{tabular}

Fuente: Elaboración propia a partir de información de Scopus.

De este modo, se observa como 14 revistas concentran el 44,6\% de las publicaciones y acumulan el 51,1\% de las citaciones, mientras el 55,4\% restante de la producción científica se distribuye en 110 revistas que aglomeran el 48,9\% de las citaciones, lo cual refleja la dispersión que respecto a este campo de estudio existe en la producción científica en revistas de impacto mundial. Vale la pena señalar como 7 de las 14 revistas con mayor número de publicaciones se caracterizan por ser del Reino Unido, concentrando cerca del $23 \%$ de la producción científica relacionada. Adicionalmente, se debe mencionar que las cinco revistas con mayor cantidad de publicaciones agrupan el $29 \%$ de la producción científica y el 34,2\% de las citaciones en este campo de estudio, estas revistas son: Australian Journal of Environmental Education (Reino Unido), Sustainability (Suiza), International Journal of Sustainability in Higher Education (Reino Unido), Journal of Cleaner Production (Países Bajos) y Journal of Environmental Studies and Sciences (Estados Unidos). 
En otro sentido, se destaca que el $41 \%$ de la producción científica relacionadas con la sostenibilidad universitaria con enfoque en la educación ambiental se encuentra en el cuartil correspondiente al 50\% de las revistas con el indicador SJR más alto, es decir, revistas en Q2. Las publicaciones en revistas en Q3 representan el 23\%; seguidamente se encuentran las revistas en el primer y cuarto cuartil que agrupan el $19 \%$ y $12 \%$ de las publicaciones, respectivamente. Se debe señalar que solo el $4 \%$ de las publicaciones identificadas se encuentran en revistas cuya cobertura fue descontinuada en Scopus, por lo tanto, no se ubican en ninguno de los cuartiles.

\section{Redes de Colaboración}

Los datos bibliográficos de las publicaciones científicas relevantes en la investigación de la sostenibilidad universitaria con enfoque en la educación ambiental, con base en la construcción de mapas bibliométricos con el software VOSviewer, permite revelar e identificar científicamente las principales redes de colaboración entre autores, organizaciones (universidades, institutos, centros de investigación, entre otros) y países. En concreto, a partir del análisis de co-autoría, se evidencia como las 231 publicaciones identificadas registran una suma total de 589 autores (ver Figura 2), asociados a 436 organizaciones (ver Figura 3) de 69 países (ver Figura 4).

En primer lugar, en la Figura 2, se visualizan los 18 autores con al menos dos publicaciones, agrupados en 12 clúster de autores fuertemente relacionados. Se resaltan los siete autores conectados por la co-autoría de cuatro publicaciones: Furnari M; Shepard K; Strack M; Barkmann J; Koch S; Sundawati L y Bögeholz S. Adicionalmente, a pesar de no estar conectados, entre los autores con mayor número de publicaciones, se destacan: Tilbury D, Fien J; Sherren K; y Shriberg M.

Figura 2. Red de autores con investigación relacionada con la sostenibilidad universitaria con enfoque en la educación ambiental. 1984-2017.

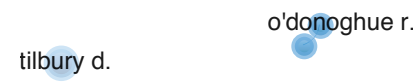

erhabor n.i.

america c.

fien $\mathrm{j}$.

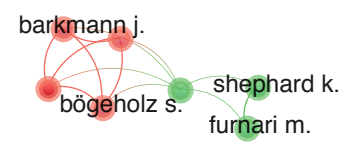

lemons j.

shriberg $\mathrm{m}$.

sherren $\mathrm{k}$.

ballantyne $r$ kopnina $\mathrm{h}$. 
Figura 3. Red de instituciones con investigación relacionada con la sostenibilidad universitaria con enfoque en la educación ambiental. 1984-2017.

$$
\begin{aligned}
& \text { albrecht-von-haller-institute } \\
& \text { university of queensland, aust departamento de psicologia evo }
\end{aligned}
$$

department of civil and enviro

charles sturt university nsw,

air quality program, washingto

departamento de biologia, univ

rmit university, australia

canterbury christ church unive department of educational scie

faculty of environmental manag

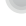

bello horizonte escazú, san jo

griffith university, brisbane,

$$
\text { av. centenario, } 303 \text { bairro sao }
$$

institute of ecology and biodi michigan state university, eas curriculum for environment and department of educational mana australian centre for environm desarrollo regional, colegio d macquarie university, australi

$$
\text { griffith university, australia }
$$

Fuente: Elaboración propia a partir de información de Scopus.

Figura 4. Red de países con investigación relacionada con la sostenibilidad universitaria con enfoque en la educación ambiental. 1984-2017.

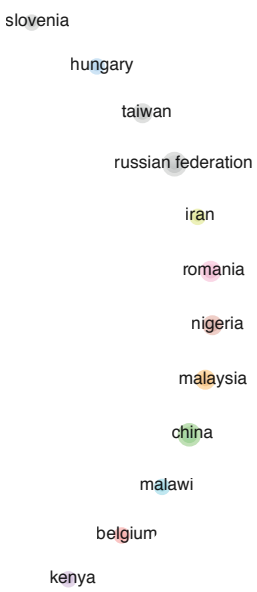

Fuente: Elaboración propia a partir de información de Scopus. 
En segundo lugar, en la Figura 3, se observan las 436 organizaciones con al menos una publicación científica relacionada con la sostenibilidad y la educación ambiental y en la universidad, evidenciando la reducida concentración de la colaboración entre los autores de diferentes organizaciones. Sin embargo, se destacan las organizaciones con mayor numero publicaciones: University of Queensland (Australia), RMIT University (Australia), Macquarie University (Australia), Griffith University (Australia) y Department of Environment and Conservation (Australia). Vale la pena mencionar que solo 11 organizaciones se encuentran conectadas entre sí por la publicaciones de sus autores.

En tercer lugar, como se observa en la Figura 4, se identifican 40 países con al menos dos publicaciones, que conforman 22 clúster de países. Se destacan los cinco países involucrados en más de 10 publicaciones: Estados Unidos, Australia, Reino Unido, España y Suráfrica. De igual manera, a partir del número de países agrupados, se pueden señalar los ocho principales clústeres de países con estrecha relación: (1) Alemania, Indonesia, Japón y Polonia; (2) Ecuador, Países Bajos, Palestina y España; (3) Italia, Noruega, Tailandia y Zambia; (4) Argentina, Canadá y Estados Unidos; (5) Australia, Chile y México; (6) Finlandia, Grecia y Suecia; (7) Nueva Zelanda y Turquía; (8) Suráfrica y Reino Unido.

\section{Influencia y Visibilidad}

En el análisis de la producción científica, del mismo modo que la contribución científica de un autor puede reflejarse en la cantidad de publicaciones, la influencia de un investigador puede representarse mediante la cantidad de citaciones registradas por sus publicaciones, mientras que la relación de citas/publicaciones muestra la influencia promedio o visibilidad de las publicaciones de un investigador (Zhu \& Wang, 2018).

Por lo que, como complemento al análisis de co-autoría realizado y cuyos resultados se presentaron en el sub-apartado anterior, a continuación, se presentan los resultados de un análisis de citaciones que pretende aproximarse a la influencia y visibilidad de los autores, revistas y países en este campo temático. De esta manera, en la Tabla 2 se ordenan los 20 autores que han realizado las mayores contribuciones a la investigación científica sobre la educación ambiental y la sostenibilidad en la universidad, a partir de: el número de publicaciones, el número de citas y la relación citas/publicaciones.

Por un lado, en términos del número de publicaciones, entre el total de 589 autores se identificaron 571 autores con solo una publicación y 18 autores con dos publicaciones. Así, el autor con mayor número de publicaciones es Tilbury D. con cuatro documentos publicados sobre este tema, seguido por autores que contribuyeron con tres publicaciones: Fien J., Sherren K. y Shriberg M. En otro sentido, en términos del número de citas, se identificaron 405 autores que registran al menos una citación, mientras que solo 14 autores registraron al menos 50 citas.

De esta manera, el investigador más influyente es Thomas I., a partir del número de citas, cuya única publicación en este campo de investigación ha sido citada 119 veces; seguido por tres autores que registran 68 citas para su única publicación: Lee J.W.C., Lim S. y Ramayah T. Mientras que, a partir de la relación citas/publicaciones, se observa como los autores que registran mayores valores de influencia promedio se caracterizan por haber publicado únicamente un documento, en su orden: Thomas I., Lee J.W.C., 
Lim S., Ramayah T., Bogner F.X., Fröhlich G., Liefländer A.K., Schultz P.W., Childs A., Corney G. y Summers M.

Vale la pena resaltar como, a pesar de registrar una sola publicación en este tema, los siguientes autores se ubican entre los autores con mayor cantidad de citas: Bogner F.X., Fröhlich G., Liefländer A.K., Schultz P.W., Childs A., Corney G., Summers M. y Nolet V. En este sentido, con excepción de Tilbury D. y Fien J., los autores con el mayor número de publicaciones no registran las mayores frecuencias de citas, evidenciando en la baja influencia promedio de dichos autores en la producción científica relacionada. También se identifican cuatro autores que, aunque no registran los mayores valores en Influencia Promedio en el conjunto total de autores, registran la mayor influencia promedio entre los autores con más de una publicación en este tema: Fien J., Furnari M., Shephard K. y Tilbury D.

Tabla 2.

Autores con mayor influencia y visibilidad en la investigación relacionada con la sostenibilidad universitaria con enfoque en la educación ambiental, en términos de documentos y citaciones. 1984-2017.

\begin{tabular}{|c|c|c|c|c|c|c|}
\hline \multirow{2}{*}{ Autores } & \multicolumn{2}{|c|}{ Documentos } & \multicolumn{2}{|c|}{ Citas } & \multicolumn{2}{|c|}{$\begin{array}{l}\text { Relación } \\
\text { Citas/Documentos }\end{array}$} \\
\hline & $\mathbf{N}^{\circ}$ & Ranking & $\mathbf{N}^{\circ}$ & Ranking & $\mathbf{N}^{\circ}$ & Ranking \\
\hline Thomas I. & 1 & 19 & 119 & 1 & 119.0 & 1 \\
\hline Lee J.W.C & 1 & 20 & 68 & 2 & 68.0 & 2 \\
\hline Lim S. & 1 & 21 & 68 & 3 & 68.0 & 3 \\
\hline Ramayah T. & 1 & 22 & 68 & 4 & 68.0 & 4 \\
\hline Bogner F.X. & 1 & 23 & 58 & 5 & 58.0 & 5 \\
\hline Fröhlich G. & 1 & 24 & 58 & 6 & 58.0 & 6 \\
\hline Liefländer A.K. & 1 & 25 & 58 & 7 & 58.0 & 7 \\
\hline Schultz P.W. & 1 & 26 & 58 & 8 & 58.0 & 8 \\
\hline Childs A. & 1 & 27 & 54 & 10 & 54.0 & 9 \\
\hline Corney G. & 1 & 28 & 54 & 11 & 54.0 & 10 \\
\hline Fien J. & 3 & 2 & 50 & 14 & 16.7 & 51 \\
\hline Furnari M. & 2 & 5 & 31 & 21 & 15.5 & 55 \\
\hline Shephard K. & 2 & 6 & 31 & 22 & 15.5 & 56 \\
\hline Tilbury D. & 4 & 1 & 56 & 9 & 14.0 & 57 \\
\hline Shallcross T. & 2 & 7 & 16 & 57 & 8.0 & 138 \\
\hline Ballantyne R. & 2 & 8 & 14 & 61 & 7.0 & 142 \\
\hline O'donoghue R. & 2 & 9 & 14 & 62 & 7.0 & 143 \\
\hline Strack M. & 2 & 10 & 14 & 63 & 7.0 & 144 \\
\hline Sherren K. & 3 & 3 & 16 & 55 & 5.3 & 196 \\
\hline Shriberg M. & 3 & 4 & 16 & 56 & 5.3 & 197 \\
\hline
\end{tabular}

Fuente: Elaboración propia a partir de información de Scopus. 
En la Tabla 3, se enumeran las 18 revistas con mayor influencia en la investigación científica sobre sostenibilidad y educación ambiental en la universidad, a partir de la identificación de las principales revistas en relación con: el número de publicaciones, el número de citas y la relación citas/documentos. Al considerar las revistas con mayor número de citas, se identifican seis revistas que se ubican entre las 10 revistas con mayor número de publicaciones: Environmental Education Research (Reino Unido), International Journal of Sustainability in Higher Education (Reino Unido), Environmental Management (Alemania), International Research in Geographical and Environmental Education (Reino Unido), Journal of Cleaner Production (Paises Bajos) y Australian Journal of Environmental Education (Reino Unido).

Mientras que entre las 10 revistas con mayor influencia promedio, con excepción de Environmental Education Research (Reino Unido) e International Journal of Sustainability in Higher Education (Reino Unido), no se encuentran las revistas con mayor cantidad de publicaciones en este campo temático. Al considerar el país de origen de las revistas, resulta interesante observar como el 72,2\% de las revistas con mayor influencia son del Reino Unido y Países Bajos. De igual manera, resulta notable la alta calidad de las publicaciones en las 18 revistas con mayor influencia en este campo temático, destacándose como el 94,4\% de estas revistas se ubican en el primer y segundo cuartil de sus respectivas categorías temáticas según el SCImago Journal \& Country Rank (2017).

Tabla 3.

Revistas con mayor influencia y visibilidad en la investigación relacionada con la sostenibilidad universitaria con enfoque en la educación ambiental, en términos de documentos y citaciones. 1984-2017.

\begin{tabular}{|c|c|c|c|c|c|c|}
\hline \multirow{2}{*}{ Revistas (país) } & \multicolumn{2}{|c|}{ Documentos } & \multicolumn{2}{|c|}{ Citas } & \multicolumn{2}{|c|}{$\begin{array}{c}\text { Relación } \\
\text { Citas/Documentos }\end{array}$} \\
\hline & $\mathrm{N}^{\circ}$ & Ranking & $\mathrm{N}^{\circ}$ & Ranking & $\mathrm{N}^{\circ}$ & Ranking \\
\hline $\begin{array}{l}\text { Educational Research } \\
\text { (Reino Unido) }\end{array}$ & 1 & 32 & 54 & 7 & 54.0 & 1 \\
\hline $\begin{array}{l}\text { Journal of Environmental } \\
\text { Management (Estados } \\
\text { Unidos) }\end{array}$ & 2 & 16 & 99 & 4 & 49.5 & 2 \\
\hline $\begin{array}{l}\text { Trends in Ecology and } \\
\text { Evolution (Países Bajos) }\end{array}$ & 1 & 33 & 34 & 13 & 34.0 & 3 \\
\hline $\begin{array}{l}\text { Journal of Environmental } \\
\text { Education (Reino Unido) }\end{array}$ & 1 & 34 & 29 & 15 & 29.0 & 4 \\
\hline $\begin{array}{l}\text { Teachers College Record } \\
\text { (Reino Unido) }\end{array}$ & 2 & 17 & 56 & 6 & 28.0 & 5 \\
\hline $\begin{array}{l}\text { Resources, Conservation } \\
\text { and Recycling (Países Bajos) }\end{array}$ & 2 & 18 & 51 & 8 & 25.5 & 6 \\
\hline
\end{tabular}

Fuente: Elaboración propia a partir de información de Scopus. 
Tabla 3.

Revistas con mayor influencia y visibilidad en la investigación relacionada con la sostenibilidad universitaria con enfoque en la educación ambiental, en términos de documentos y citaciones. 1984-2017.

\begin{tabular}{|c|c|c|c|c|c|c|}
\hline \multirow{2}{*}{ Revistas (país) } & \multicolumn{2}{|c|}{ Documentos } & \multicolumn{2}{|c|}{ Citas } & \multicolumn{2}{|c|}{$\begin{array}{l}\text { Relación } \\
\text { Citas/Documentos }\end{array}$} \\
\hline & $\mathbf{N}^{\circ}$ & Ranking & $\mathbf{N}^{\circ}$ & Ranking & $\mathbf{N}^{\circ}$ & Ranking \\
\hline $\begin{array}{l}\text { Environmental Education } \\
\text { Research (Reino Unido) }\end{array}$ & 5 & 6 & 116 & 3 & 23.2 & 7 \\
\hline $\begin{array}{l}\text { Ecological Modelling } \\
\text { (Paises Bajos) }\end{array}$ & 1 & 35 & 23 & 17 & 23.0 & 8 \\
\hline $\begin{array}{l}\text { Ecological Indicators } \\
\text { (Países Bajos) }\end{array}$ & 1 & 36 & 21 & 19 & 21.0 & 9 \\
\hline $\begin{array}{l}\text { International Journal of } \\
\text { Sustainability in Higher } \\
\text { Education (Reino Unido) }\end{array}$ & 10 & 3 & 185 & 1 & 18.5 & 10 \\
\hline $\begin{array}{l}\text { Environmental } \\
\text { Management (Alemania) }\end{array}$ & 4 & 9 & 43 & 9 & 10.8 & 17 \\
\hline $\begin{array}{l}\text { Jorunal of Geography in } \\
\text { Higher Education } \\
\text { (Reino Unido) }\end{array}$ & 4 & 10 & 34 & 12 & 8.5 & 24 \\
\hline $\begin{array}{l}\text { International Research in } \\
\text { Geographical and } \\
\text { Environmental Education } \\
\text { (Reino Unido) }\end{array}$ & 5 & 7 & 42 & 10 & 8.4 & 25 \\
\hline $\begin{array}{l}\text { Journal of Cleaner } \\
\text { Production (Países Bajos) }\end{array}$ & 10 & 4 & 81 & 5 & 8.1 & 26 \\
\hline $\begin{array}{l}\text { Australian Journal of } \\
\text { Environmental Education } \\
\text { (Reino Unido) }\end{array}$ & 25 & 1 & 173 & 2 & 6.9 & 33 \\
\hline Sustainability (Suiza) & 12 & 2 & 34 & 11 & 2.8 & 51 \\
\hline $\begin{array}{l}\text { Journal of Environmental } \\
\text { Studies and Sciences } \\
\text { (Estados Unidos) }\end{array}$ & 10 & 5 & 28 & 16 & 2.8 & 52 \\
\hline $\begin{array}{l}\text { Environmental Engineering } \\
\text { and Management Journal } \\
\text { (Rumania) }\end{array}$ & 5 & 8 & 7 & 35 & 1.4 & 63 \\
\hline
\end{tabular}

Fuente: Elaboración propia a partir de información de Scopus. 
Ahora bien, a partir de los 69 países en donde se ubican las organizaciones de afiliación de los autores de las publicaciones relacionadas con la sostenibilidad y la educación ambiental en la universidad, se identificaron los 16 países con la mayor influencia (ver Tabla 4), con base en su: número de publicaciones, numero de citaciones y relación citas/documentos. De esta manera, entre los 10 países con mayor influencia promedio se encuentran cuatro países que concentran un número significativo de publicaciones (Australia, Alemania, Reino Unido y Canadá) y seis países que agrupan un mínimo número de publicaciones (Myanmar, Malasia, Jamaica, Argentina, Bélgica y Ecuador).

Por otra parte, en los 10 primeros países con mayor número de publicaciones se identifican seis países que registran una influencia promedio regular, como: Estados Unidos, España, Nueva Zelanda, Turquía, Sudáfrica y Brasil. En este punto, resulta interesante el caso de Estados Unidos que, a pesar de ubicarse como el país con mayor número de publicaciones y citas, registra una influencia promedio regular y se sitúa en el puesto 11 entre los 18 países con mayor influencia.

Tabla 4

Países con mayor influencia y visibilidad en la investigación relacionada con la sostenibilidad universitaria con enfoque en la educación ambiental, en términos de documentos y citaciones. 1984-2017.

\begin{tabular}{|c|c|c|c|c|c|c|}
\hline \multirow{2}{*}{ Países } & \multicolumn{2}{|c|}{ Documentos } & \multicolumn{2}{|c|}{ Citas } & \multicolumn{2}{|c|}{$\begin{array}{c}\text { Relación } \\
\text { Citas/Documentos }\end{array}$} \\
\hline & $\mathrm{N}^{\circ}$ & Ranking & $\mathrm{N}^{\circ}$ & Ranking & $\mathrm{N}^{\circ}$ & Ranking \\
\hline Myanmar & 1 & 60 & 31 & 12 & 31.0 & 1 \\
\hline Malasia & 3 & 24 & 71 & 7 & 23.7 & 2 \\
\hline Jamaica & 1 & 53 & 16 & 21 & 16.0 & 3 \\
\hline Australia & 31 & 2 & 357 & 2 & 11.5 & 4 \\
\hline Argentina & 3 & 20 & 34 & 11 & 11.3 & 5 \\
\hline Bélgica & 2 & 28 & 22 & 17 & 11.0 & 6 \\
\hline Alemania & 7 & 9 & 75 & 5 & 10.7 & 7 \\
\hline Reino Unido & 15 & 3 & 145 & 3 & 9.7 & 8 \\
\hline Canadá & 9 & 7 & 75 & 4 & 8.3 & 9 \\
\hline Ecuador & 2 & 30 & 16 & 20 & 8.0 & 10 \\
\hline Estados Unidos & 49 & 1 & 359 & 1 & 7.3 & 11 \\
\hline España & 11 & 4 & 72 & 6 & 6.5 & 14 \\
\hline Nueva Zelanda & 7 & 10 & 44 & 9 & 6.3 & 15 \\
\hline Turquía & 9 & 8 & 47 & 8 & 5.2 & 19 \\
\hline Sudáfrica & 10 & 5 & 38 & 10 & 3.8 & 30 \\
\hline Brasil & 9 & 6 & 15 & 22 & 1.7 & 43 \\
\hline
\end{tabular}

Fuente: Elaboración propia a partir de información de Scopus. 


\section{Tendencias Temáticas}

El análisis previo de las publicaciones científicas, realizado y presentado anteriormente en este documento, permite vislumbrar la complejidad y variedad de los hallazgos en la investigación científica relacionada con la sostenibilidad y la educación ambiental en la universidad. A partir de lo descrito previamente, se realizó un análisis de co-ocurrencia de palabras claves con el propósito de identificar los términos o grupos de términos relacionados entre las publicaciones científicas en este campo de investigación.

En efecto, a pesar que se identificaron un total de 1.232 palabras claves descriptoras de las publicaciones seleccionadas, en el análisis de co-ocurrencia se evidencia la existencia de 59 palabras claves que ocurrieron por lo menos cinco veces y se encuentran relacionados entre sí, que se encuentran agrupadas en cuatro clúster. Por lo que, en la construcción de los mapas bibliométricos de las palabras claves se incluyeron solo estos 59 términos y en la Figura 5 se aprecian los términos de las palabras claves indicados por su etiqueta y también por un círculo.

De esta manera, se observa la existencia de cuatro clúster de palabras claves conectados y una significativa dispersión de la mayoría de los términos de los clúster, entre los que se pueden mencionar: (1) Inicialmente, en el clúster identificado con el color rojo se encuentran, entre otros, los términos Canada, Conservation, Decisión Making, Ecology, Education Policy, Education Program, Educational Development, Enviromental Engineering, Enviromental Management, Enviromental Planning, Enviromental Protection, Enviromental Research, Enviromental Studies y Forestry; (2) seguidamente en el clúster identificado con el color verde agrupan los terminos Education For Sustainable Develoment, Enviromental Education, Enviromental Issue, Eurasia, Higher Education, Knoledgment, Learning, Local Participation, Natural Resource, Perception, Resource Management, Sustainability y Training; (3) posteriormente, en el clúster identificado con el color azul están conectados los términos Attitude, Economics, Education, Environment, Enviromental Sustainability, Planning, Recycling, Societies and Institutions, Students, Sustainable Development, University, University Students y Waste Management; y (4) finalmente, en el clúster con menor cantidad de palabras claves e ilustrado con el color amarillo se identifican los términos Climate Change, Curricula, Curriculum, Engineering, Engineering Education, Environmental Impact, Sustainability Education y Teaching.

Ahora bien, excluyendo los términos incluidos en las expresiones de búsqueda detallada en la metodología de este trabajo, los términos que registraron mayor fuerza de relación y enlaces de co-ocurrencias más fuertes son: Higher Education, Student, Curriculum, Learning, Environmental Protection, Teaching, United States, Educational Development, Environment y Environmental Management.

Por otra parte, en la Figura 6 se aprecian significativas diferencias temporales en la ocurrencia de los términos claves de las publicaciones científicas. A partir de lo anterior, resulta notable como en el periodo comprendido por los años anteriores al 2008, los clúster con mayor concentración de publicaciones se encuentran vinculados a los términos como: Environmental Protection, Euroasia, Ecology, Environment, Enviromental Planning, International Cooperation y Enviromental Engineering. Mientras que en los años posteriores a 2008, los clústeres con mayor concentración de publicaciones están vinculados a los términos: Student, Engineering, Education For Sustainable Develoment, Curricula, Sustainability, Curriculum, Learning y Educational Development. 
Figura 5. Red de palabras claves en la investigación relacionada con la sostenibilidad universitaria con enfoque en la educación ambiental, visualización de clúster. 1984-2017.

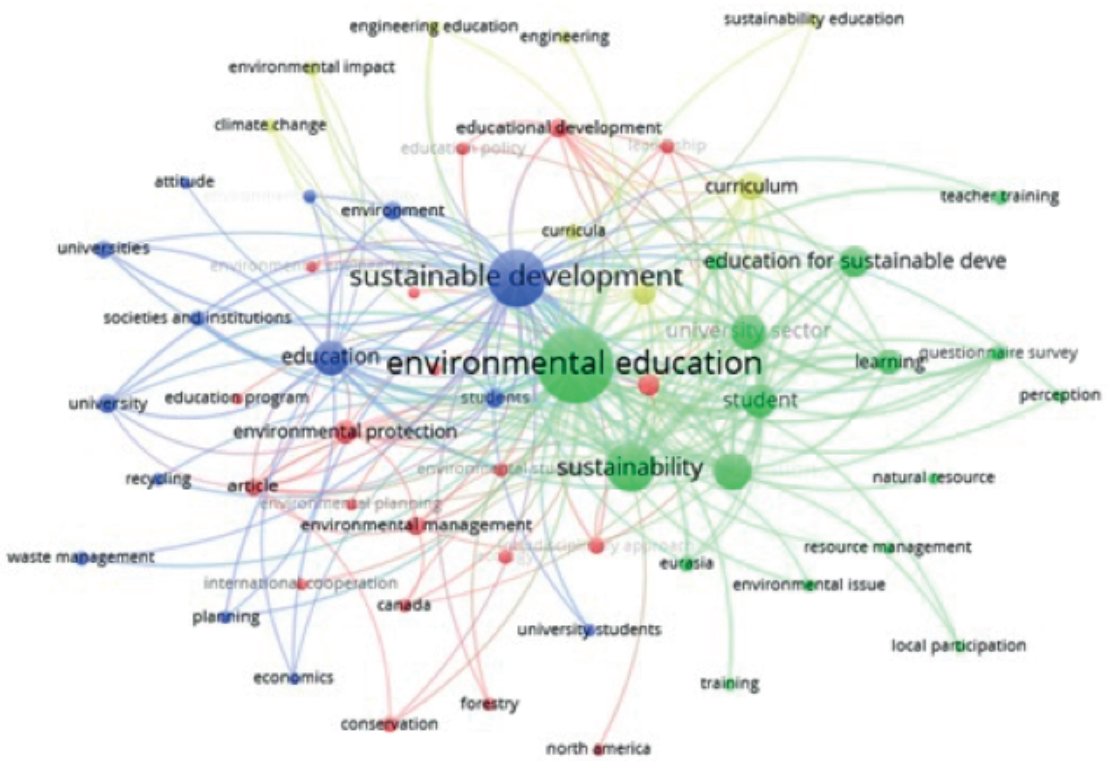

Fuente: Elaboración propia a partir de información de Scopus.

Figura 6. Red de palabras claves en la investigación relacionada con la sostenibilidad universitaria con enfoque en la educación ambiental, visualización temporal. 1984-2017.

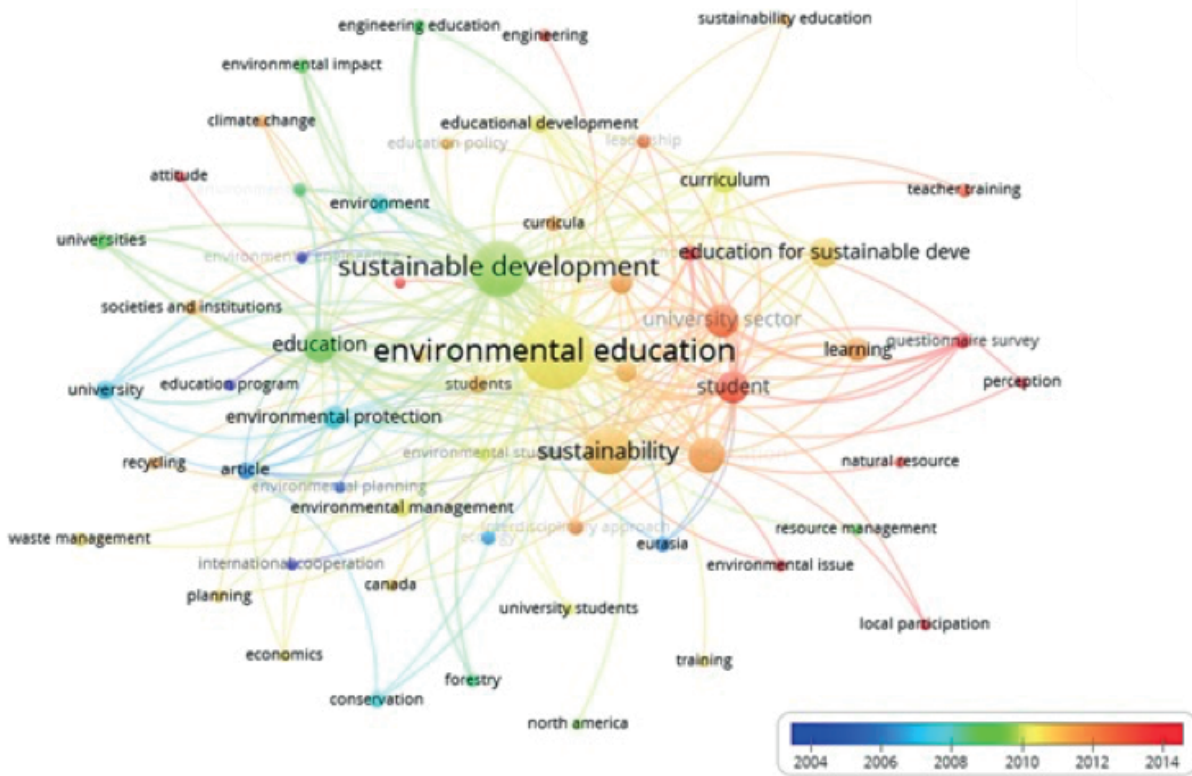

Fuente: Elaboración propia a partir de información de Scopus. 
Seguidamente, en la Figura 7 se observa como el Clúster 1 y el Clúster 2, que concentran el 62,7\% de las 59 principales palabras claves, registran el 66,6\% de las ocurrencias de las palabras claves agrupadas. Por su parte, el 37,3\% restante de las palabras claves se distribuye en los Clúster 3 y Clúster 4, los cuales registran el 33,4\% de las sumatoria de las ocurrencias de estos términos. Se debe mencionar que las principales tendencias temáticas de la investigación científico sobre la sostenibilidad y la educación ambiental en la universidad, se relacionan con las palabras claves que registran una relación más estrecha y mayor co-ocurrencia entre las demás palabras claves.

En este orden de ideas, a pesar de agrupar la segunda mayor cantidad de palabras claves, el Clúster 2 se posiciona como el clúster con mayor concentración de ocurrencias de los términos y se encuentra estrechamente relacionado con las palabras claves Student, Learning y Education for Sustainable Development. De igual manera, resulta interesante observar la estrecha relación de las palabras claves de mayor co-ocurrencia del Clúster 2 con los principales términos concentrados en el Clúster 4: Climate Change, Curricula y Curriculum. En otro sentido, se aprecia que las palabras claves del Clúster 1 registrar una importante asociación de los términos del Clúster 3.

En efecto, a partir del análisis descriptivo y de co-ocurrencia realizado en este trabajo, fueron identificados los principales enfoques de la producción científica relacionada con la sostenibilidad y la educación ambiental en la universidad. Así, con el fin de proporcionar una visión general de la investigación relacionada, a continuación se presenta una breve descripción analítica de las tendencias temáticas de investigación identificados en este campo de estudio. Es importante aclarar que la agrupación de la producción científica no es mutuamente excluyente y solo intenta presentar la fuerte interrelación de las temáticas abordadas en las publicaciones. 
Figura 7. Frecuencia de ocurrencia de palabras claves en la investigación relacionada con la sostenibilidad universitaria con enfoque en la educación ambiental, por clúster. 1984-2017.

Número de ocurrencias

0100200300400500600700800

\begin{tabular}{|c|c|c|c|c|c|c|}
\hline \multirow{16}{*}{ 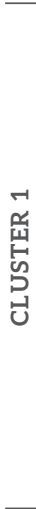 } & Environmental Protection & 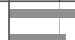 & 130 & & & \multirow{48}{*}{689} \\
\hline & Educational Development & $\square$ & 96 & & & \\
\hline & & 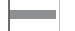 & 92 & & & \\
\hline & Environmental Management & 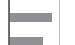 & $\begin{array}{l}86 \\
65\end{array}$ & & & \\
\hline & Education Program & 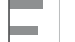 & $\begin{array}{l}65 \\
55\end{array}$ & & & \\
\hline & Lulation regram & $\square$ & 55 & & & \\
\hline & Leadership & 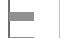 & 52 & & & \\
\hline & & 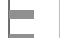 & 52 & & & \\
\hline & Forestry & 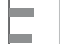 & 52 & & & \\
\hline & Conservation & 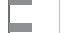 & $\begin{array}{l}40 \\
47\end{array}$ & & & \\
\hline & Conservation & E & 47 & & & \\
\hline & Environmental Engineering & $=$ & 46 & & & \\
\hline & & E & 46 & & & \\
\hline & Environmental Planning & E & 45 & & & \\
\hline & 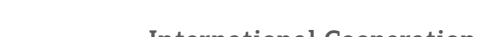 & E & $\begin{array}{l}43 \\
36\end{array}$ & & & \\
\hline & International Cooperation & E & $\begin{array}{l}30 \\
28\end{array}$ & & & \\
\hline \multirow{14}{*}{ 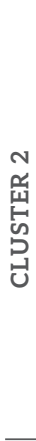 } & Environmental Education & & & & & \\
\hline & University Sector & & $=$ & 260 & \multirow{31}{*}{473} & \\
\hline & 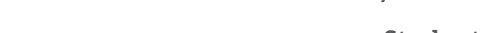 & & & 237 & & \\
\hline & Student & 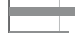 & & 236 & & \\
\hline & & 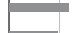 & & 118 & & \\
\hline & Education For Sustainable Development & 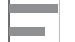 & & $\begin{array}{l}98 \\
66\end{array}$ & & \\
\hline & Knowledge & 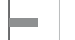 & & 58 & & \\
\hline & & 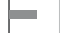 & & 53 & & \\
\hline & Environmental Issue & $E$ & & 45 & & \\
\hline & Training & E & & 39 & & \\
\hline & Training & E & & $\begin{array}{l}37 \\
30\end{array}$ & & \\
\hline & Teacher Training & E & & 30 & & \\
\hline & & E & & 28 & & \\
\hline & kesource management & P & & & & \\
\hline \multirow{12}{*}{ 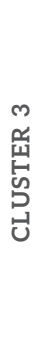 } & Education & & 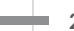 & & & \\
\hline & & & & & & \\
\hline & University & 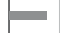 & & & & \\
\hline & & 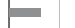 & & & & \\
\hline & Universities & 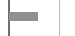 & & & & \\
\hline & & 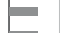 & & & & \\
\hline & Environmental Sustainbility & - & & & & \\
\hline & Recycling & E & & & & \\
\hline & & 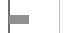 & & & & \\
\hline & Waste Management & E & & & & \\
\hline & (2) & $=$ & & & & \\
\hline & Attitude & $=$ & & & & \\
\hline \multirow{6}{*}{ 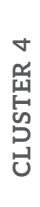 } & Teaching & 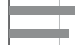 & $\begin{array}{l}156 \\
117\end{array}$ & & & \\
\hline & & 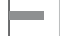 & 68 & & & \\
\hline & Environmental Impact & E & $\begin{array}{l}51 \\
36\end{array}$ & & & \\
\hline & Engineering Education & 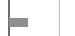 & 34 & & & \\
\hline & Sustainability Education & & 32 & & & \\
\hline & Sustainability Education & H & 22 & & & \\
\hline
\end{tabular}

Fuente: Elaboración propia a partir de información de Scopus. 
De esta manera, los principales términos se encuentran agrupados en dos grandes grupos de términos significativamente relacionados: (1) Student, Curriculum, Learning y Teaching; (2) Education, Environmental Protection, Educational Development y Environmental Management. Estas palabras claves permiten inferir la importancia y relevancia de dos corrientes o tendencias de la investigación científico sobre la educación ambiental y la sostenibilidad en la universidad: (1) La educación ambiental para la sostenibilidad y (2) el rol de la universidad en relación con la educación ambiental y sostenibilidad de la sociedad.

Por un lado, en primer lugar, en la tendencia de investigación sobre la educación ambiental para la sostenibilidad desde la universidad, se incluyen dos importantes líneas de estudio: la integración de la sostenibilidad en los currículos educativos y la adaptación del proceso de aprendizaje y enseñanza de los estudiantes universitarios. En coherencia con otros autores (Collazo Expósito \& Geli de Ciurana, 2017; Karatzoglou, 2012; Lozano et al., 2014; Schmitt Figueiró \& Raufflet, 2015; Toscano-Hernández et al., 2018), se evidencia la necesidad de impulsar cambios fundamentales en la concepción del proceso educativo, como: fomentar la relación con la sociedad en general y las organizaciones sociales en particular, incorporación de proyectos de trabajo interdisciplinarios, reforzar la colaboración con entidades locales, fortalecer los esfuerzos para abordar el estudio y analisis de problemas de carácter local y global, favorecer la coherencia entre los postulados teóricos y las acciones organizacionales.

Por otro lado, en segundo lugar, la tendencia que aborda el rol de la universidad en relación con la educación ambiental y sostenibilidad de la sociedad abarca diversas temáticas como: iniciativas de protección ambiental (Lozano et al., 2014), el desarrollo educativo (Schmitt Figueiró \& Raufflet, 2015), la colaboración con los distintos actores de la sociedad (Collazo Expósito \& Geli de Ciurana, 2017; Lozano et al., 2014), la incorporación de trabajos de carácter interdisciplinar (Collazo Expósito \& Geli de Ciurana, 2017) y la gestión ambiental de la universidad (Lozano et al., 2014).

En general, resulta especialmente útil evidenciar como la sostenibilidad universitaria con enfoque en la educación ambiental se ubica como un campo de estudio con una importancia significativa en la gestión de la educación superior, al valorar su potenciales aportes para abordar apremiantes problemas sociales. En concreto, el foco de la investigación relacionada con la sostenibilidad y la educación ambiental se encuentra en la perspectiva del aprendizaje y la educación de los estudiantes para el desarrollo sostenible.

\section{CONCLUSIONES}

\section{Principales tendencias de la investigación}

Uno de los principales resultados evidenciados en este trabajo, a partir del análisis descriptivo, es el importante interés generado en este campo de estudio, reflejado en el importante incremento registrado en el número anual de publicaciones, al registrar un aumento del $1400 \%$ en el número de publicaciones anuales entre 1984 y 2017 . En lo referente a la dispersión, que respecto a este campo de estudio existe en la producción científica en revistas de impacto mundial, las principales 14 revistas concentran el 44,6\% de las publicaciones y acumulan el 51,1\% de las citaciones. 
Por otra parte, se evidencia como las 231 publicaciones identificadas registran un total de 589 autores, asociados a 436 organizaciones de 69 países. Entre los 18 autores con al menos dos publicaciones, resaltan los siete autores que se encuentran conectados por la co-autoría de cuatro publicaciones: Furnari M; Shepard K; Strack M; Barkmann J; Koch S; Sundawati L y Bögeholz S. En relación a la influencia y visibilidad, a partir la relación citas/publicaciones, se observa como los autores que registran mayores valores se caracterizan por haber publicado únicamente un documento, en su orden: Thomas I., Lee J.W.C., Lim S., Ramayah T., Bogner F.X., Fröhlich G., Liefländer A.K., Schultz P.W., Childs A., Corney G. y Summers M.

En lo referente a las organizaciones, se evidencia la reducida concentración de la colaboración entre los autores de diferentes organizaciones, destacándose las cinco organizaciones con mayor número de publicaciones: University of Queensland (Australia), RMIT University (Australia), Macquarie University (Australia), Griffith University (Australia) y Department of Environment and Conservation (Australia). Entre los 10 países con mayor influencia promedio se encuentran cuatro países que concentran un número significativo de publicaciones (Australia, Alemania, Reino Unido y Canadá) y seis países que agrupan un mínimo número de publicaciones (Myanmar, Malasia, Jamaica, Argentina, Bélgica y Ecuador).

En general, los hallazgos de este trabajo permiten inferir la importancia y relevancia de dos corrientes o tendencias de la investigación científica sobre la educación ambiental y la sostenibilidad en la universidad: (1) La educación ambiental para la sostenibilidad y (2) el rol de la universidad en relación con la educación ambiental y sostenibilidad de la sociedad.

\section{Implicaciones para la sostenibilidad universitaria}

Este trabajo pretende ampliar la comprensión de los académicos, investigadores y líderes universitarios sobre la educación ambiental y la sostenibilidad en la gestión universitaria. En concreto, desde un enfoque práctico, los resultados de este trabajo aportan valiosas contribuciones, relacionadas con: (1) el desarrollo de la investigación científica relacionada, (2) la formación de futuros profesionales, así como a (3) la gestión de la sostenibilidad en la universidad.

En primer lugar, los académicos e investigadores pueden acceder a los avances científicos de mayor influencia e impacto, identificar potenciales temáticas de estudios o vacíos que faciliten la formulación de nuevas preguntas de investigación. En segundo lugar, los estudiantes y profesores de programas académicos de pregrado y posgrados podrían utilizar los resultados de esta investigación para facilitar la comprensión del potencial que brinda la educación ambiental y la sostenibilidad en la universidad, así como presentar los aspectos metodológicos asociados a un estudio bibliométrico de la producción científica. En tercer lugar, para los gerentes y líderes en el sector de educación superior que diseñan estrategias organizacionales esta información es vital, dado que los resultados brindan información de las características y evolución temporal de la investigación científica de mayor relevancia mundial, para considerar las implicaciones relacionadas en que contribuyen a la optimización de la gestión universitaria a partir de los principios de la sostenibilidad. 


\section{Potenciales direcciones de investigación futura}

Los resultados de este trabajo permiten no solo identificar las tendencias de investigación abordadas hasta la actualidad, sino que además evidencia los vacíos existentes. Frente a los crecientes retos de la universidad como actor crucial de la sociedad, a pesar a que la diversidad de la producción científica es una señal positiva de los avances de la sostenibilidad en la educación superior (Toscano-Hernández et al., 2018), el desarrollo de la investigación relacionada con la educación ambiental y sostenibilidad debe continuar e inevitablemente debe considerar no solo a los estudiantes universitarios sino a los demás stakeholders de la universidad como empresas, sector público, profesores, entre otros.

\section{Limitaciones del estudio}

Los resultados de este trabajo complementan los aportes realizados por estudios bibliométricos previos (Lazzarini \& Pérez-Foguet, 2018; Lazzarini et al., 2018; Pipere et al., 2015; Schmitt Figueiró \& Raufflet, 2015; Toscano-Hernández et al., 2018), a partir de una metodología que se esforzó por desarrollar un procedimiento exhaustivo e incluyente. No obstante, dado que la identificación y selección de la producción científica fue realizada a partir de unos criterios específicos, en la praxis los hallazgos no representa la totalidad y variedad de los aportes científicos en este campo de investigación, y deben ser interpretados con prudencia. En efecto, para futuros trabajos se sugiere ampliar el alcance de la búsqueda, en lo que se refiere a la cantidad de bases de datos y tipos de documentos incluidos.

\section{REFERENCIAS BIBLIOGRÁFICAS}

Alba, D., Alonso, I. y Benayas, J. (2011). La Agenda 21 educativa en la universidad. En A. Melendro, M., Murga, M. A. y Cano (Ed.), IDEAS. Iniciativas de educación ambiental para la sostenibilidad. Madrid (España): Universidad Nacional de Educación a Distancia.

Alba Hidalgo, D. (2017). Hacia una fundamentación de la sostenibilidad en la educación superior. Revista Iberoamericana de Educación, 73, 15-34.

Bartels, E. M. (2013). How to perform a systematic search. Best Practice and Research: Clinical Rheumatology, 27(2), 295-306. http://doi.org/10.1016/j.berh.2013.02.001

Collazo Expósito, L., \& Geli de Ciurana, A. (2017). Avanzar en la educación para la sostenibilidad. Combinación de metodologías para trabajar el pensamiento crítico y autónomo, la reflexión y la capacidad de transformación del sistema. Revista Iberoamericana de Educación, 73, 131-154.

Falagas, M. E., Pitsouni, E. I., Malietzis, G. a, \& Pappas, G. (2008). Comparison of PubMed, Scopus, Web of Science, and Google Scholar: strengths and weaknesses. The FASEB journal: official publication of the Federation of American Societies for Experimental Biology, 22(2), 338-42. http://doi.org/10.1096/fj.07-9492LSF

Gaudiano, E. J. G., Meira-Cartea, P. A., \& Martínez-Fernández, Y. C. N. (2015). Sustentabilidad y Universidad: Retos, ritos y posibles rutas. Revista de la Educacion Superior, 44(175), 69-93. http://doi.org/10.1016/j.resu.2015.09.002

Herrera-Llamas, J. (2014). La universidad desde la perspectiva del 2020 : El futuro del mercado universitario en Colombia. Panorama Económico, 21, 231-242. 
Karatzoglou, B. (2012). An in-depth literature review of the evolving roles and contributions of universities to Education for Sustainable Development. Journal of Cleaner Production, 49(june 2013), 44-53. http://doi.org/10.1016/j.jclepro.2012.07.043

Lazzarini, B., \& Pérez-Foguet, A. (2018). Profiling research of the engineering academics who successfully promote education in Sustainable Human Development. Journal of Cleaner Production, 172, 4239-4253. http://doi.org/10.1016/j.jclepro.2017.08.234

Lazzarini, B., Pérez-Foguet, A., \& Boni, A. (2018). Key characteristics of academics promoting Sustainable Human Development within engineering studies. Journal of Cleaner Production, 188, 237-252. http://doi.org/10.1016/J.JCLEPRO.2018.03.270

Linnenluecke, M. K., \& Griffiths, A. (2013). Firms and sustainability: Mapping the intellectual origins and structure of the corporate sustainability field. Global Environmental Change, 23(1), 382-391. http://doi.org/10.1016/j.gloenvcha.2012.07.007

Lozano, R., Ceulemans, K., Alonso-Almeida, M., Huisingh, D., Lozano, F. J., Waas, T., ... Hug, J. (2014). A review of commitment and implementation of sustainable development in higher education : results from a worldwide survey. Journal of Cleaner Production, 108, 1-18. http://doi.org/10.1016/j.jclepro.2014.09.048

Lozano, R., Lukman, R., Lozano, F. J., Huisingh, D., \& Lambrechts, W. (2013). Declarations for sustainability in higher education: Becoming better leaders, through addressing the university system. Journal of Cleaner Production, 48, 10-19. http://doi.org/10.1016/j. jclepro.2011.10.006

Moher, D., Liberati, A., Tetzlaff, J., Altman, D. G., Altman, D., Antes, G., ... Tugwell, P. (2009). Preferred reporting items for systematic reviews and meta-analyses: The PRISMA statement. PLoS Medicine, 6(7). http://doi.org/10.1371/journal.pmed.1000097

Perianes-Rodriguez, A., Waltman, L., \& Van Eck, N. J. (2016). Constructing bibliometric networks: A comparison between full and fractional counting. Journal of Informetrics, 10(4), 11781195. http://doi.org/10.1016/j.joi.2016.10.006

Pipere, A., Veisson, M., \& Salite, I. (2015). Developing Research in Teacher Education for Sustainability: UN DESD via the Journal of Teacher Education for Sustainability. Journal of Teacher Education for Sustainability, 17(2), 5-43. http://doi.org/10.1515/jtes-2015-0009

Schmitt Figueiró, P., \& Raufflet, E. (2015). Sustainability in higher education : a systematic review with focus on management education. Journal of Cleaner Production journal, 106, 22-33. http://doi.org/10.1016/j.jclepro.2015.04.118

Tilbury, D. (2010). Sustainability in the DNA of the university. Sustainable Mediterranean, 63, 9-13.

Toscano-Hernández, A., Álvarez-González, L. I., \& Sanzo-Pérez, M. J. (2018). Tendencias globales de la investigación científica relacionada con la sostenibilidad en la Universidad: Un análisis bibliométrico 2008-2017. En E. Älvarez-Arregui, S. García Granda, J. R. Obeso Suárez, J. J. Coz Díaz, F. Lombo Brugos, M. Mitre Aranda, ... V. Cañal Fernández (Eds.), Universidad, Investigación y Conocimiento: Comprensión e intervención en una sociedad compleja. Oviedo (España): Servicio de Publicaciones de la Universidad de Oviedo.

Tranfield, D., Denyer, D., \& Smart, P. (2003). Towards a methodology for developing evidenceinformed management knowledge by means of systematic review. British Journal of Management, 14(3), 207-222. http://doi.org/10.1111/1467-8551.00375

UNESCO. (2014). Hoja de ruta para la ejecución del Programa de acción mundial de Educación para el Desarrollo Sostenible. Paris (France). 
Van Eck, N. J., \& Waltman, L. (2010). Software survey: VOSviewer, a computer program for bibliometric mapping. Scientometrics, 84(2), 523-538. http://doi.org/10.1007/s11192-0090146-3

Van Eck, N. J., \& Waltman, L. (2016). VOSviewer Manual (Manual for VOSviewer version 1.6.5). Universiteit Leiden. Recuperado a partir de http://www.vosviewer.com/

Vergara-Arrieta, J. J., \& Álvarez-Carval, Y. (2017). Una mirada a los métodos de valoración de calidad ambiental desde una visión contable. Panorama Económico, 24, 221-238.

Vilches, A., \& Gil, D. (2012). La educación para la sostenibilidad en la Universidad: el reto de la formación del profesorado. Profesorado. Revista de Currículum y Formación de Profesorado, 16(2), 25-43.

World Bank. (2017). Higher Education for Development: An Evaluation of the World Bank Group's Support. Washington (EEUU). Recuperado a partir de http://documents.worldbank.org

Zhu, W., \& Wang, Z. (2018). The Collaborative Networks and Thematic Trends of Research on Purchasing and Supply Management for Environmental Sustainability : A Bibliometric Review. Sustainability, 10(5), 2-28. http://doi.org/10.3390/su10051510 